\title{
A Deterministic Model of the Vertical Jump: IMPLICATIONS FOR TRAINING
}

\author{
DANiel J. HaM, ${ }^{1}$ WADE L. Knez, ${ }^{1,2}$ AND WARren B. Young ${ }^{1}$ \\ ${ }^{1}$ University of Ballarat, School of Human Movement and Sports Science, Mt. Helen, Victoria, Australia; ${ }^{2}$ Institute \\ of Sport and Exercise Science, James Cook University, Cairns, Queensland, Australia.
}

\begin{abstract}
Ham, D.J., W.L. Knez, and W.B. Young. A deterministic model of the vertical jump: implications for training. $J$. Strength Cond. Res. 21(3):967-972. 2007.-Increasing vertical jump height is a critical component for performance enhancement in many sports. It takes on a number of different forms and conditions, including double and single legged jumps and stationary and run-up jumps. In an attempt to understand the factors that influence vertical jump performance, an extensive analysis was undertaken using the deterministic model. Once identified, practical training strategies enabling improvement in these factors were elucidated. Our analysis showed that a successful vertical jump performance was the result of a complex interplay of run-up speed, reactive strength, concentric action power of the take-off leg(s), hip flexors, shoulders, body position, body mass, and take-off time. Of special interest, our analysis showed that the concentric action power of the legs was the critical factor affecting stationary double leg vertical jumps, whereas reactive strength was the critical component for a single leg jump from a run-up.
\end{abstract}

KEY WORDS. muscular power, performance optimization, training methodology

\section{INTRODUCTION}

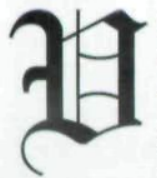

ertical jumping (VJ) is an important feature of many sports, and increasing the height to which an athlete can reach may be advantageous to sport performance (10). Much research has been conducted on this activity, which has provided insights into possible strategies for training (6, 23,32 ). However, previous studies have been limited to standing double legged take-offs, where contact times are $>500 \mathrm{~ms}$, whereas some sports involve run-ups and single leg take-offs with an associated take-off time that can be $<200 \mathrm{~ms}$. Subsequently, it is necessary to determine what factors influence performance in these specific jumps, so that further insights into appropriate training practices may be elucidated. Therefore, the purpose of this article is to develop a detailed deterministic model to indicate the factors that govern VJ performance and discuss practical training strategies and technique adaptations that may be adopted to improve jumping performance. Such a methodical approach is yet to be completed in detail and is intended to assist the strength and conditioning professional by providing a map of factors that contribute to optimal performance of a skill, allowing them to focus training methods on identified weaknesses. It should be noted that specific training exercises and coaching methods are beyond the scope of this paper, as this is seen as the responsibility of coaches and strength and conditioning professionals.

\section{Deterministic Model}

In this study, a form of qualitative analysis, not dissimilar to the mechanical method outlined by Hay and Reid
(10), has been used to develop a deterministic model for the VJ. This method involves systematically examining the performance of a skill to determine what contributory factors may be improved to enhance overall performance (12). This is achieved by identifying the bottom line factors, which are the determinants of the skill and the performance in their most purest form. For example, jump height is determined by take-off height, flight height of the center of gravity, and reach height. Reach height is in turn determined by body position and limb lengths, which are not determined by any other factors and are therefore termed bottom line factors. This type of analysis aims to supplement whatever experience the sport scientist or coach might have and to channel or direct the analysis in a logical, systematic fashion (12), eliminating the risk of overlooking influential factors. In this study, bottom line factors from a detailed deterministic model were analyzed from a biomechanical and strength and conditioning perspective and will be used as a precursor for practical training strategies and technique adaptations for the VJ.

Vertical Jump. The VJ is frequently used in a variety of sports and has a number of components that require specific abilities that are not necessarily interrelated. The VJ can take the broad form of a single leg jump for reach height (basketball lay-up), a single leg jump for clearance height (high jump), a double leg jump from a stationary position (volleyball block), and a double leg jump with a run-up (volleyball spike). In this analysis, a deterministic model was developed to cover all of these types of jumps and to explore specific practical training applications and technique adaptations. Inter-jump-type differences are also discussed in detail, and further recommendations for these techniques are given.

Generic Jump Deterministic Model. The model depicted in Figure 1 represents a generic deterministic model adapted from the model developed by Hay and Reid (10). Some factors are not relevant for every jump type. Indeed, neither run-up speed nor hip flexor power is relevant for a standing countermovement jump, but because they are important to a single leg run-up jump, they have been included in the model.

\section{Research Design}

In this study, the pathway from VJ height is followed along the model branch to the extremities, or bottom line factors, which have been highlighted in Figure 1. These bottom line factors represent the determinants of VJ performance in their simplest form-contributing factors that are not contributed to by any other factors. Furthermore, these bottom line factors have been divided into 2 categories, with the exception of limb lengths, which have been left out because of their unchangeable nature. The first category is specifically relevant to strength and con- 


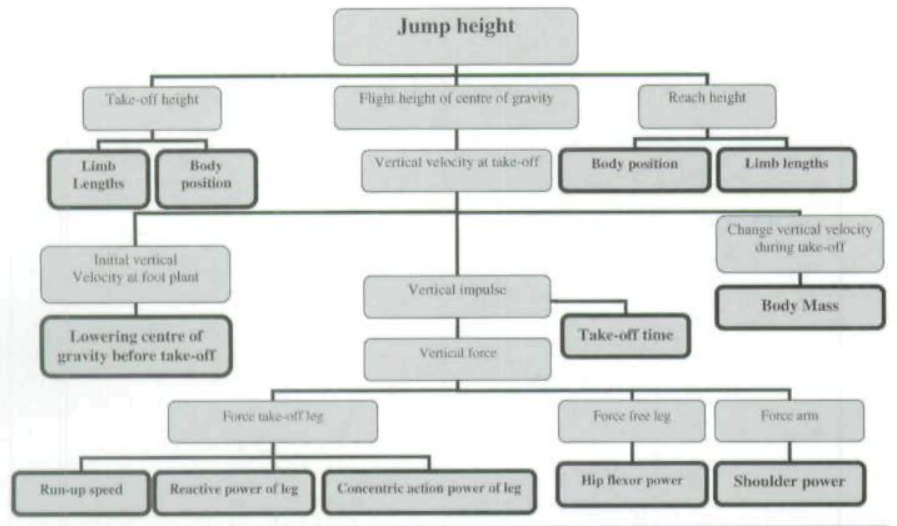

FIGURE 1. Deterministic model of performance for a vertical jump (bottom line factors are in bold).

ditioning, and the second represents important areas for technique adaptation and body size considerations.

\section{Category 1-STRength AND CoNDITIONING}

\section{Run-up Speed}

For sports such as high jump, it is believed that a fast approach run improves the height of the jump (6); however, it has been shown that athletes have an individual optimum run-up speed (31). Indeed, the faster the approach velocity, the more the jumper is dependent on the strength of the take-off leg (28). If an approach run is very fast, and the take-off leg is not strong enough, these joints will be forced to flex excessively, or "buckle," during the first half of the take-off phase, and this will weaken the subsequent extension of the leg (6). The ability to resist this buckling of the leg during the eccentric phase of the take-off is considered vital for successful jumping (30), and jumpers must therefore be able to tolerate high stretch loads to achieve optimum jump heights (31). Moreover, the jumpers who used the fastest run-ups achieved the greatest vertical velocities at take-off (6), which suggests that better performing jumpers were those with greatest dynamic strength. Therefore, greater dynamic strength will allow an athlete to use a faster run-up speed and subsequently achieve a greater take-off velocity and jump higher.

\section{Training Applications}

Optimal technique will involve using the fastest approach run possible without reaching the threshold for buckling. If optimum run-up speed is slow, but close to buckling threshold, appropriate strength training should be carried out to enable the athlete to resist excessive flexion during the first half of the take-off phase. Because buckling occurs in the first phase of the foot plant, and the muscle contraction in this phase is eccentric, appropriate training should focus on the legs ability to resist large amounts of force during eccentric contraction.

\section{Reactive Power of the Leg}

Reactive strength is the ability to quickly switch from the eccentric to concentric phase in a stretch shortening cycle (SSC) (31) and can add to power production during the concentric action of the takeoff leg(s) that will ultimately increase jump height. It is thought to be a relatively independent strength quality that is of importance in jumps involving short contact times and large take-off forces (27). Indeed, even long SSCs ( $>0.250$ seconds) and short
SSCs $(<0.250$ seconds) have been found to be independent skills, and therefore, it is possible for an athlete to excel in one and not the other (30). Reactive strength correlates best with short SSCs, such as run-up jumps, where contact time is low and force is high (32). The stretch component of the SSC refers to the eccentric muscle action, whereas the shortening component refers to the concentric muscle action; elastic energy is stored in the tendomuscular system during the eccentric phase and released during the concentric phase (25).

Studies examining different characteristics of the SSC have shown increases in force and power production by decreasing the duration of the amortization phase (electromechanical delay between eccentric and concentric actions), a direct result of an increase in reactive strength, and increasing the speed of eccentric action (25). Recent studies have shown that the series elastic elements/muscle-tendon complex in the lower leg (below the knee) were able to store and release elastic energy even during a vertical squat jump that consisted of solely concentric muscle contraction (3, 19). Furthermore, increasing tension placed on these elements/structures through greater muscle contraction increased the amount of elastic energy that could be stored and released. Considering that much higher intrinsic muscle forces are able to be developed during fast eccentric contractions (15), it is not surprising that reactive strength is an important factor in jumps that involve high eccentric loads. Indeed, it has been found that fast eccentric contractions and small amortization phases, associated with a short SSC, increases the amount of stored energy that can be released during the subsequent concentric phase of jumps. This is especially true in fast twitch fibers (2).

Reactive strength is a very specific skill that is characterized by the high forces and low contact times indicative of jumps involving a run-up. It is linked with the effective functioning of a short SSC $(<0.250$ seconds $)$ and can be improved by increasing the amount of energy that is stored in the tendomuscular system during the eccentric phase and ensuring maximum release of this energy by decreasing the length of the amortization phase.

\section{Training Applications}

To increase reactive strength and optimize the use of stored energy for power production, plyometric exercises, such as depth jumps with a focus on maximum height and minimum contact time, should be used (25), because they focus heavily on the rapid change from eccentric to concentric contractions. Depth jumps have been shown to be more effective in increasing power output, force production, and jumping performance than other VJ tests such as countermovement jumps (25). Quality of training is important for the development of reactive strength, because an increase or decrease from an individual's optimal depth jump height has been found to decrease performance and therefore improperly overloading the functioning of the SSC (25). Furthermore, specificity is a crucial factor, because research has shown that eccentric strength can be improved by 3 times as much through eccentric training than concentric training (13). Furthermore, eccentric training protects muscle from injury during subsequent eccentric contractions $(5,17,22,26)$. Therefore, preceding plyometric training with specific eccentric training may decrease the risk of injury and decrease the time needed for recovery because of decreased muscle damage. 


\section{Concentric Action Power of the Legs}

Increasing the ability to produce power $(P=F \times d / t)$ during the concentric action of the take-off phase of a VJ takes on 2 main pathways: (a) strengthening specific muscle groups and (b) alteration of specific neuromuscular parameters (23). A recent simulation study found that strengthening of the knee flexor muscle group and increasing the neuromuscular property $F_{\max }$ had the largest effect on VJ performance compared with increasing plantar flexors or hip extensors and increasing maximum velocity $\left(V_{\max }\right)$ or activation capacity, respectively (23). Interestingly, strengthening the plantar flexors, hip extensors, and knee flexors and increasing $F_{\max }, V_{\max }$, and activation capacity simultaneously corresponded to a greater increase in jump height than the sum of the individual muscles or parameters (23). This study was, however, based solely on the squat jump, and therefore, its application to jumps requiring a run-up is limited. Indeed, this is especially pertinent when considering that a poor correlation exists between $F_{\max }$ /weight and both a double-leg countermovement jump with no run-up and a single-leg jump from a run-up (32). Conversely, Jaric et al. (14) found a significant correlation between both rate of force development (RFD), which is similar to $V_{\max }$ and $F_{\max }$ of different muscle groups, and counter-movement jump. However, no significant correlations were found between RFD and $F_{\max }$, reiterating the independence of strength qualities. Despite these inconsistencies, the contribution of $F_{\max }$ to jumps involving a SSC is not to be underestimated, because an 80 -ms isometric contraction separates the stretch and shortening phases (9). In support of this, a number of training studies have shown that the gains in VJ performance are similar for heavy resistance strength and high-velocity SSC training methods (32). Although it is likely that increases in $F_{\max }$ of between 20 and $30 \%$ after strength training programs lasting between 2 and 6 months are possible (8, 13, 18, 24), any weight gains caused by increased muscle cross-sectional area may counter the effects of increased $F_{\max }$ on VJ performance. Although an increase in weight is a negative side affect of hypertrophy training, an increase in muscle size should not always be avoided. Increases in the crosssectional area of vastus lateralis are paralleled by increases in muscle fibre pennation angle, which places the muscle fiber in a mechanically superior position and increases the muscles capacity to produce force (1).

Although it is clearly advantageous to increase the concentric action power of the legs, it is important to note that, for the benefits of increased strength and power in individual muscles to be fully realized in VJ performance, reoptimization of the muscle activation patterns is required $(4,23)$.

\section{Training Applications}

Temporarily discounting the effect of body weight on VJ height, it would seem that increasing the muscle crosssectional area of the ankle plantar flexors, knee extensors, and hip extensors to increase $F_{\max }$ and, in some cases, increase the pennation angle for mechanical superiority should be a primary focus of training for squat jump like movements. Although the role of $F_{\max }$ in jumps involving a SSC is not as significant as it is in a squat jump, it is likely that this strength quality is of at least some relevance because of the presence of isometric contractions in the SSC. To achieve optimum improvement in concentric action power of the legs, all relevant muscle groups and strength qualities must be improved in similar proportions. The most important training application when attempting to increase concentric action power of the legs and subsequent improvement in VJ height is that muscle activation patterns must be reoptimized for transfer of increased strength qualities to increased VJ performance. Reoptimization can be achieved through incorporation of jumping movements throughout the training program so that the athlete can learn how to use the strengthened muscles.

\section{Hip Flexor Power}

The effect of hip flexor power on VJ performance is relevant for sports involving a single leg jump, such as high jump; however, because this type of jump is prevalent in many sports, it is an important factor to consider. In a study on single and double leg jumps, all subjects were able to jump $75 \%$ of their standing double leg VJ height with right and left legs separately with the use of both an arm and free leg swing (33). Although others have recorded single leg jumps that were only $58.5 \%$ of double leg scores, the use of arms and the free leg was restricted (29). The large discrepancy between these values may be explained by the benefits derived from the swing of the free leg. Although the effect of the free leg was quite apparent during a stationary single leg jump, its influence on VJ height during the much more sport-related run-up jump is not as clear. In a study investigating the relative contribution of the free limbs to whole body vertical momentum, the free limbs were judged to contribute $7.1 \%$; however, it was suggested that the arms provided a greater contribution to this value than the lead leg (21).

\section{Training Applications}

Although it is likely that hip-flexors of the free leg contribute to performance in a single leg run-up jump, the contribution is likely to be fairly small. In sports such as high jump, where every edge for performance is critical, it is recommended that athletes spend some time developing the hip flexors; however, there are other trainable factors that will elicit far greater improvements in performance. For this reason, development of hip flexor power should not be a primary focus of training for increased VJ performance. In contrast, the free leg's contribution to take-off height through elevation should not be underestimated.

\section{Shoulder Power}

Several studies have undergone investigation into the influence of arm swing during different types of VJ. Arm swing has been found to increase jump height by $10-27 \%$, depending on the type of jump and type of athletes selected in the protocol $(7,11,16,20,27)$. There have been a number of reasons given for the apparent increase in VJ height associated with an arm swing. The fact that arm swing increases vertical ground reaction impulse (VGRI) is widely accepted; however, theories on how this is accomplished vary. Kinematic changes occurring with an arm swing are also thought to contribute to increased VJ performance. Unlike VGRI, the contribution of kinematic changes to increased VJ height is both supported (11) and refuted (16) throughout the literature.

Research has shown that arm swing increases the tension placed on each segment of the jump, allowing for improved functioning of the SSC, particularly in the leg extensors, and a greater total VGRI $(11,16)$. Although it is clear that arm swing increases VGRI, observations of 
jumpers have shown that, in most cases, the arms return to zero vertical velocity relative to the rest of the body as they approach the fully raised position before take-off, meaning the net effect on VGRI should be about zero (11). Despite the use of an arm swing contributing very little to VGRI, it can place the muscles of the take-off leg(s) under faster eccentric conditions and slower concentric conditions depending on the period of the take-off phase (6), which is advantageous when considering the forcevelocity relationship of muscle contraction (11). However, if the athlete's take-off leg is already near the threshold for buckling, stronger arm actions could take the athlete beyond the optimum and result in a suboptimal jump performance (6). The upward/backward swing of the arms partially offsets the downward acceleration of the nonarm body mass, causing a decrease in unweighting during the countermovement (11). When the muscles crossing the hip and knee are in the most advantageous position to exert vertical ground reaction force (VGRF), the upward acceleration of the arms creates a downward force at the shoulders on the rest of the body, slowing the contraction of the large quadriceps and gluteal muscles to velocities at which they can exert more force (11). By the time the arms decelerate near the end of their swing, which makes the quadriceps and gluteals contract at a more rapid speed and consequently diminishing force generation capability, the knees and hips are almost fully extended, and the muscles around them are not in a position to generate much positive VGRF (11). Arm swing may also contribute to an increased VJ height through an increase in pre-take-off total body center of mass (TBCM) displacement. Interplay between peak pre-take-off TBCM rise and increased VGRI has been suggested because of increases in VJ height after an arm swing that exceed increases in VGRI alone (11).

\section{Training Applications}

Ultimately, arm swing during a countermovement jump increases the TBCM rise; however, the interplay between kinematic changes and the increase in VGRI has yet to be quantified. Shoulder power training should be included in an exercise program with a focus of increasing VJ height. However, in sports with a single-leg jump from a run-up, such as high jump, where the leg extensors are already placed under considerable eccentric load, the extent to which shoulder power can increase jump height is ultimately reliant on the eccentric strength of the athlete's legs and should therefore be reflected in training.

\section{Category 2-Skills Coach}

\section{Body Position-Take-off and Reach Height}

The capacity to jump and reach for height is an important attribute in many sports (32) and can be improved through a number of methods. Alteration of body position during both the pre-take-off, which has been discussed previously, and post-take-off phases can significantly improve VJ performance. During the post-take-off phase of a VJ-and-reach test an athlete will thrust the nontouching arm downward while reaching up with the other arm (11). Lowering $1 \mathrm{arm}$ and fully extending the legs will lower the position of TBCM within the body and hence allow the raised arm to be further from the TBCM and higher from the ground. This redistribution of body mass during the flight phase of the VJ can only be enabled through body position alterations during the pre-take-off phase. Raising of the free limbs (the arms for a double leg jump and the arms and non-take-off leg for a single leg jump) causes a positive vertical shift in the TBCM within the body and is an imperative prerequisite for post-take-off internal TBCM lowering.

\section{Training Applications}

Although the positive effects of increasing pre-take-off TBCM have yet to be quantified, it is clearly beneficial to VJ height (11). Raising the limbs during the pre-take-off phase and lowering of all but $1 \mathrm{arm}$ (where possible) during the post-take-off phase also increases VJ reach height. Although these benefits may be small, training this technique is likely to be far less time consuming than making physiological adaptations and should be included in training where applicable. Obviously the extent to which body position can be used to increase the TBCM depends on the requirements of the jump within the sporting context. Indeed, a block in volleyball will not allow for these adjustments.

\section{Lowering Center of Mass Before Take-off}

Some investigators have proposed that maintaining a low center of mass at the end of the approach run also improves the height of a VJ (6). The effect of lowering the center of mass before take-off was found by Dapena et al. (4) to be very similar in effect to increasing run-up speed. If the approach is very low and the take-off leg is not strong enough, the joints of the take-off leg will be forced to flex excessively during the first half of the take-off phase, and this will weaken the subsequent extension of the leg (6). Therefore, the extent to which an athlete is able to lower his/her center of gravity at the end of the approach run without forcing the take-off leg to buckle is dependent on the strength capabilities of the take-off leg. These findings refer specifically to single leg jumps from a run-up, and it is unclear whether they will be practically applicable for double leg jumps.

\section{Training Applications}

Because run-up speed and lowering of the center of mass before take-off both rely on strength capabilities of the take-off leg, it would seem logical that there be an optimum combination of run-up speed and center of mass height for individual athletes. If the optimum combination of run-up speed and center of mass height is slow and high, strength qualities, particularly eccentric, must be worked on, because it means the take-off leg is weak.

\section{Body Mass}

Although hypertrophy of the leg muscles has been shown to improve VJ performance, it is important to note that any gain in mass without an associated increase in strength could negatively influence performance. Jumping for maximum height is a powerful action, dominated by fast twitch fiber contraction, therefore it is important to avoid excessive hypertrophy of slow twitch fibers, especially in jumps involving low contact times. The capacity to produce force during a maximal voluntary contraction is determined by the cross-sectional area and activation ability of the relevant muscles (29); it is therefore important to accompany gains in muscle size with maintenance or increase in activation percentage of the associated muscles.

\section{Training Applications}

Non-force-producing tissue is useless for the VJ and should be minimized. Diet should be monitored along 
with training to ensure a low body fat percentage. Training programs should aim to maximize the activation percentage of all relevant muscles, especially after hypertrophy training. Furthermore, hypertrophy of irrelevant muscle groups and slow twitch fibers should be avoided.

\section{Take-off Time}

Although, in theory, a greater take-off time would allow for a greater impulse (force $\times$ time $=$ impulse) in relation to reactive strength, a short contact time is imperative for storing and reusing elastic energy during the SSC in jumps with a run-up, particularly if the run-up is fast. Noteworthy, contact time increases from single leg jumps with a run-up to double leg countermovement and squat jumps, the relative importance of the reuse of elastic energy decreases $(3,19)$, and the importance of the muscles ability to produce force during the concentric muscle phase increases.

\section{Training Applications}

Take-off times during plyometric exercises are imperative to specific training of different strength qualities and muscle functions. As previously stated, long and short SSCs are independent muscle functions, and training for 1 type will not necessarily improve performance in the other. Subsequently, take-off times used in training should mimic those in competition.

\section{Practical Applications}

\section{Training Applications for Double Leg Jumps}

For double leg VJs from a stationary position, the relative involvement of the SSC is small, and training should therefore focus on increasing the concentric action power of the ankle plantar flexors, knee extensors, and hip extensors simultaneously, with knee extensors being the major contributor. This should be achieved through weight training that increases maximum force through increased cross-sectional area.

\section{Training Applications for Single Leg Jumps}

The ability to jump with a single leg take-off from a runup can be improved by increasing the threshold for buckling that effectively changes body position and alters the height of the center of mass. The limiting physiologic factor is the ability of the take-off leg to resist and store large amounts of force during the eccentric phase and the effective reuse of this stored energy in the subsequent concentric phase. This can be achieved through specific eccentric training coupled with plyometric exercises, such as depth jumps that optimize the use of stored energy through a focus on the rapid change form eccentric to concentric contraction conditions. Also, preceding plyometric training with specific eccentric training may decrease the risk of injury and increase recovery time. Further improvements can be achieved by raising and subsequent lowering of the arms and free leg during the pre- and post-take-off phases, respectively. This increases VJ height by increasing the pre-take-off TBCM rise and increasing reach height.

The VJ is a physiologically and biomechanically complex movement. It relies on the interplay of many physical properties that combine and alter the magnitude of their contribution to affect different jump types. Training aimed at increasing VJ height needs to be highly specific to the type of jump required in athletic competition. Through proper strength and power training and tech- nique adaptations, VJ performance can be improved substantially.

\section{REFERENCES}

1. Aagaard, P., J.L. Anderson, P. Dyhre-Poulsen, A. Leffere, A. WAGNER, S.P. Magnusson, J. Hal.kJaer-Kristensen, and E.B. Simonsen. A mechanism for increased contractile strength of human pinnate muscle in response to strength training: changes in muscle architecture. J. Phy. siol. 534:613-623. 2001.

2. AURA, O., AND J.L. VitTASALO. Biomechanical characteristics of jumping. Int. J. Sport Biomechanics, 5:89-98. 1989.

3. BoBbERT, M.F. Dependence of human squat jump performance on the series elastic compliance of the triceps surae: A simulation study. J. Exp. Biol. 204:533-542. 2001

4. BREED, R.V.P., AND W.B. YounG. The effect of a resistance training programme on the grab, track and swing starts in swimming. J. Sports Sci. 21:213-220. 2003.

5. Clarkson, P.M., AND Tremblay I. Exercise-induced muscle damage, repair, and adaptation in humans. J. Appl. Physiol. 65:1-6. 1988.

6. DAPENA, J., C. MCDONALD, AND J. CAPPAERT. A regression analysis of high jumping technique. Int. J. Sport Biomechanics. 6:246-261. 1990.

7. DAviES, B.N., AND K.G. JonEs. An analysis of the performance of male students in the vertical and standing long jump tests and the contribution of arm swing. J. Human Movement Studies. 24:25-38. 1993.

8. DAVIES, C.T.M., AND K. Young. Effects of training at 30 and $100 \%$ maximal isometric force (MVC) on the contractile properties of the triceps surae in man. J. Physiol. 336:22-23. 1983.

9. Gollhofer, A., V. Strojnik, W. RAPP, AND L. Schweizer. Behavior of triceps surae muscle-tendon complex in different jump conditions. Eur. J. Appl. Physiol. Occupat. Physiol. 64:283-291. 1992.

10. HAKKINEN, K. Changes in physical fitness profile in female basketball players during the competitive season including explosive type strength training. J. Sports Med. Phys. Fitness. 33:19-26, 1993.

11. Harman, E.A., M.T. Rosenstein, P.N. Frykman, and R.M. Rosenstein. The effects of arms and countermovements on vertical jumping. Med. Sci. Sports Exerc. 22:825-833. 1990.

12. Hay, J.G., AND J.G. Rem. Anatomy, Mechanics, and Human Motion. Englewood Cliffs, NJ: Prentice Hall, 1988.

13. Higbie, E.J., K.J. Cureton, G.L. Warren, and B.M. Prior. Effects of concentric and eccentric training on muscle strength, cross-sectional area, and neural activation. J. Appl. Physiol. 81:2173-2181. 1996.

14. JARIC, S., D. Ristanovic, AND D,M. CorCos. The relationship between muscle kinetic parameters and kinetic variables in a complex movement. Eur. J. Appl. Physiol. 59:370-376. 1989.

15. Jonhagen, S., AND N. GunNar. Hamstring injuries in sprinters. Am. J. Sports Med. 22:262-266. 1994.

16. KHALID, W., M. Amin, AND T. BobER. The influence of the upper extremities movement on take-off in vertical jump. In: Biomechanics in Sports. V.L. Tsarouchas, J. Terauds, B.A. Gowitzke, and L.E. Holt, eds. Athens: Hellenic Sports Research Institute, 1998. pp. 375-379.

17. KOH, T. J., AND S.V. BROOKS. Lengthening contractions are not required to induace protection from contraction-induced muscle injury. Am. J. Physiol. 281:R155-R161. 2001.

18. KOMI, P.V., AND A. Bosco. Utilization of stored elastic energy in leg extensor muscles by men and women. Med. Sci. Sports Exerc. 10:261265. 1978.

19. Kurokawa, S., T. FukUnaga, and S. FuKashiro. Behavior of fascicles and tendinous structures of human gastrocnemius during vertical jumping. J. Appl. Physiol. 90:1349-1358. 2001.

20. LEES, A., AND G. BARTON. The interpretation of relative momentum data to assess the contribution of the free limbs to the generation of vertical velocity in sports activities. J. Sports Sci. 14:503-511. 1996.

21. Lees, A., J. Rojas, M. Ceperos, V. Soto, And M. Gutierrez. How the free limbs are used by elite high jumpers in generating vertical velocity. Ergonomics. 43:1622-1636. 2000.

22. McHugh, M.P., D.A.J. Connolly, R.G. Eston, and G.W. Gleim. Exercise-induced muscle damage and potential mechanisms for the repeated bout effect. Sports Med. 27:157-170. 1999.

23. NAGANO, A., AND K.G.M. GERRITSEN. Effects of neuromuscular strength training on vertical jumping performance-a computer simulation study. J. Appl. Biomechanics. 17:113-128, 2001.

24. Narichi, M.V., H. Hoppeler, B. Kayser, L. Landoni, H. ClaAsen, C. Gavardi, M. Conti, AND P. Cerretelli. Human quadriceps cross-sectional area, torque and neural activation during 6 months strength training. Acta Physiol. Scand. 157:175-186. 1996.

25. READ, M.M., AND C. CISAR. The influence of varied rest interval lengths on depth jump performance. J. Strength Cond. Res. 15:279-283. 2001.

26. SCHWANE, J.A., AND R.B. ARMSTRONG. Effect of training on skeletal muscle injury from downhill running in rats. J. Appl. Physiol. 55:969-975. 1983. 
27. SHETTY, A.B., AND B.R. ETNYRE. Contribution of arm movement to the force components of a maximum vertical jump. J. Orthopaed. Sports Phys. Ther. 11:198-201. 1989.

28. ShibuKaWA, M.A.K., S. TADA, AND Y. HashiHARA. A biomechanical analysis of the segmental contribution to the take-off of the one-leg running jump for height. In: Biomechanics VIII. H. Matsui, K. Kobayashi, eds. Chicago: Human Kinetics, 1983. pp. 737-745.

29. VAN SOEST, A.J. M.E. ROEBROECK, P.A. HUIJING, G.J. VAN INGEN SCHENAU, AND M.F. BobBERT. A comparison of one-legged and two legged countermovement jumps. Med. Sci. Sports Exerc. 17:635-639. 1985.

30. Young, W. Specificity of jumping ability and implications for training and testing athletes. In: Proceedings of the National Coaching Conference. Canberra, Australian Coaching Council, 1994. pp. 217-221.
31. YounG, W. Specificity of strength development for improving the takeoff ability in jumping events. Modern Athlete Coach. 33:3-8. 1995.

32. Young, W., G. Wilson, AND C. BYrne. Relationship between strength qualities and performance in standing and run-up vertical jumps. $J$. Sports Med. Phys. Fitness. 39:285-293. 1999.

33. Young, W.B., C. MCDONALD, AND M.A. Flowers. Validity of double- and single-leg vertical jumps as tests of leg extensor muscle function. $J$. Strength Cond. Res. 15:6-11. 2001.

Address correspondence to Dr. Daniel J. Ham, d.ham@ ballarat.edu.au. 
Copyright of Journal of Strength \& Conditioning Research is the property of Alliance Communications Group and its content may not be copied or emailed to multiple sites or posted to a listserv without the copyright holder's express written permission. However, users may print, download, or email articles for individual use. 